\title{
Inhalative Therapie mit Dosier-Aerosolen: Fehleranalyse und Varianten der Verbesserung
}

\author{
W. Petro \\ A. Schuppenies
}

\author{
Inhalation Therapy by Dose-Inhalers: Analysis of Patients Performance \\ and Possibilities for Improvement
}

\section{Zusammenfassung}

Basis- und Bedarfstherapie obstruktiver Atemwegserkrankungen erfordern eine effiziente inhalative Applikation. Die vorliegende Arbeit befasst sich mit der Fehleranalyse bei der Handhabung von Dosier-Aerosolen, setzt diese in Beziehung zum Grad der Vorbildung in der Anwendung und prüft, welche Varianten zur Verbesserung des Wissens und Handhabungsablaufes bei der inhalativen Applikation notwendig sind. 125 Patienten mit chronisch obstruktiven Atemwegserkrankungen wurden in Hinblick auf die Anwendung von Dosier-Aerosolen untersucht und in sechs verschiedene Gruppen je nach Vorbildungsgrad eingeteilt. Die Handhabung wurde sowohl in standardisierten Einzelschritten als auch im gesamten Ablauf beurteilt. Des weiteren wurde die Effizienz eines interaktiven PC-basierten Schulungsprogramms geprüft. Die schlechteste Handhabung zeigten Patienten, die nur durch den Beipackzettel informiert waren. Patienten mit ambulanter Schulung, wie auch Patienten mit Kleingruppenschulung während der stationären Behandlung zeigten eine Verbesserung des Handhabungsstandards. Eine große Verbesserungsrate ließ sich bei zunächst Dosier-Aerosol-naiven Patienten und anschließender Schulung mittels PC-basierten interaktiven Patiententraining erzielen. Die meisten Probleme beziehen sich auf den Vorgang des Ausatmens vor der Inhalation und auf den Schritt der Inhalation. Neben der klassischen Schulung und PCbasierter Schulung ist auch der Einsatz von Dosier-AerosolPhantomen hilfreich. Die praktische Schlussfolgerung aus dieser

\section{Abstract}

Inhalation therapy in chronic obstructive airways disease requires an efficient inhalation technique. This study analyses step by step the mistakes made in the usage of different MDIs, relates these to patient information prior to the testing and examines several teaching procedures for improvement of knowledge and performance of the inhalation technique. 125 patients suffering from COPD were assigned to six different groups according to their background knowledge in the inhalation technique. The performance was assessed in standardized single steps and as overall performance. Furthermore the efficacy of an interactive pc-based-training program was evaluated. The worst performance was seen in patients who only used the suppliers medication leaflet as a guide. Patients trained in outpatient clinics as well as patients trained in small groups during an inpatient stay showed a better performance. A high improvement rate was seen in prior MDI naive patients after they had undergone the interactive pc-based training program. Most problems were detected in the application step "exhalation before inhalation" and in the actuation-inhalation step. Besides the classical and the pc-based training the use of MDI phantoms showed very good results. The practical conclusion of this study is that the ability of patients to use inhalation pharmacotherapy efficiently needs improvement. Training programs of different intensity lead to a different outcome in performance and knowledge depending on prior knowledge. Inhalation pharmacotherapy without adequate training is insufficient.

Institutsangaben

Klinik Bad Reichenhall, Fachklinik für Erkrankung der Atmungsorgane, Allergien und für Orthopädie

Anmerkung

Teilergebnisse der Dissertationsschrift „Untersuchungen zur Handhabung verschiedener inhalativer Applikationssysteme bei Patienten mit chronisch obstruktiven Atemwegserkrankungen“, A. Schuppenies, TU München, 2003

Korrespondenzadiresse

Prof. Dr. med. Wolfgang Petro · Klinik Bad Reichenhall · Fachklinik für Erkrankungen der Atmungsorgane, Allergien und für Orthopädie · Salzburger Str. 8-11 · 83435 Bad Reichenhall . E-mail: wolfgang.petro@klinik-bad-reichenhall.de

Eingang: 11. Juni $2004 \cdot$ Nach Revision alkzeptiert: 10. Dezember 2004

Bibliografie

Pneumologie 2005; 59: 316-320 @ Georg Thieme Verlag KG Stuttgart · New York DOI 10.1055/s-2004-830213

ISSN 0934-8387 
Untersuchung ist, dass Fähigkeiten und Handhabung bei inhalativer Medikamentenapplikation dringend verbesserungsbedürftig sind. Schulungsmaßnahmen unterschiedlicher Intensität führen in Abhängigkeit vom Vorbildungsgrad zu einer Verbesserung des Wissens und der Handhabung. Eine inhalative Pharmakotherapie ohne adäquate Schulung ist unzureichend.

\section{Einleitung}

Die inhalative Therapie bei obstruktiven Atemwegserkrankungen, insbesondere bei Asthma und COPD ist die Basis pharmakotherapeutischer Bemühungen [13,14]. Der Vorteil der Inhalationstherapie besteht in der direkten Deposition am Ort der Krankheit, der Bronchialschleimhaut. Somit ist die benötigte Dosis gering und dementsprechend geringer die unerwünschten Wirkungen. Diesen Vorteilen steht der Nachteil der Komplexität der inhalativen Applikation gegenüber. Bekanntermaßen haben viele Patienten erhebliche Schwierigkeiten ihr Dosier-Aerosol korrekt zu benutzen $[3-5,8,10]$. Spezielle Schulungsformen können die Applikationsfehler verbessern [5,7] oder gänzlich beseitigen [8].

Ziel der vorliegenden Untersuchung war es zu analysieren, welche Fehler Patienten mit obstruktiven Atemwegserkrankungen bei der inhalativen Applikation in Abhängigkeit vom Grad der Vorkenntnisse in der Anwendung machen und wie effizient verschiedene Varianten der Wissensverbesserung funktionieren.

\section{Methodik}

Die Untersuchungen erfolgten an 125 Patienten, 41 (32,8\%) weiblichen Geschlechts, 84 (67,2\%) männlichen Geschlechts, mit chronisch obstruktiven Atemwegserkrankungen im Rahmen eines stationären Rehabilitationsaufenthaltes an der Klinik Bad Reichenhall in Form einer prospektiven, nicht randomisierten Zufallsstichprobe. Das Alter der Patienten war mit 20 bis 80 Jahren breit gestreut. Das mittlere Alter betrug 58,5 Jahre. Es handelt sich um Patienten mit mittlerem Bildungsgrad (Haupt- und Realschulabschluss) ohne starke Beeintächtigung der physischen oder psychischen Fähigkeiten.

Folgende inhalative Applikationssysteme werden verwendet: Druckdosieraerosol (MDI), Autohalerprinzip, Pulverinhalatoren (DPI), Diskus, Turbohaler, Novolizer, Aerolizer.

Zur Beantwortung der Fragestellung wurden insgesamt 6 Patientengruppen unterschiedlicher Vorbildung hinsichtlich theoretischem Wissen und Handhabung für den Gebrauch von DosierAerosolen untersucht (Tab.1). Bei den Patienten handelt es sich ausschließlich um Arbeiter oder Rentner mit vergleichbarem Bildungsniveau. Anhand standardisierter Beobachtungsbögen erfolgte die Handhabungsbeurteilung bei allen Patientengruppen von derselben Person nach denselben Kriterien.

Um eine Vergleichbarkeit zwischen verschiedenen Applikatoren zu sichern, wurde ein standardisierter Punktescore gebildet, der

Tab. 1 Übersicht untersuchter Patientengruppen, deren Applikationsvorkenntnisse und Art der Intervention

Gruppe 1

$(n=49, d A=62,1)$

Gruppe 2

$(n=45, d A=57,2)$

Gruppe 3

$(n=22, d A=65,4)$

Gruppe 4

$(n=30, d A=65,4)$

Gruppe 5

$(n=28, d A=65,4)$

Gruppe $6 a$

$(n=23, d A=53,5)$

Gruppe $6 b$

$(n=23, d A=53,5)$
Patienten, ohne spezielle Schulung, denen von medizinischem Personal mindestens einmal die korrekte Handhabung gezeigt worden war

DA-erfahrene Patienten, die schon ein- oder mehrmals an verschiedenen Schulungen außerhalb der Klinik teilgenommen hatten

DA-naive Patienten, die sich ausschließlich mit dem Beipackzettel informiert hatten

DA-naive Patienten ohne jegliche Vorinformation oder Erfahrung

zunächst DA-naive Patienten nach Schulung auf der jeweiligen Station in Kleingruppen

DA-naive Patienten ohne Information oder Schulung.

zunächst DA-naive Patienten der Gruppe 6a nach Schulung mit dem Computerprogramm „MoreBreath“

Anmerkungen zur Tab. 1: DA = Dosieraerosol; $\mathrm{n}=$ Anzahl der Patienten; $\mathrm{dA}=$ Durchschnittsalter in Jahren; Schulung auf der Station in Kleingruppen: Die Patienten wurden vom Pflegepersonal in einer 90 Minuten dauernden Schulung unterrichtet. Im ersten Abschnitt wurden Grundlagen der Atmung und Medikamentenwirkstoffe („Schützer“, „Helfer“) erklärt. Im zweiten Abschnitt wurden Funktionsweise verschiedener Applikationssysteme und deren korrekte Handhabung erklärt und vorgeführt. Die Patienten zeigten anschließend die Handhabung an ihrem Gerät selbst. Eventuelle Fehler wurden sofort korrigiert. Schulung mit der CD-Rom „More-BREATH“. Die CD-Rom wurde in Anwesenheit eines Lehrers durchlaufen. Entsprechende Applikationssysteme können per Mouseklick ausgewählt werden. In einer Animation wird von einem Arzt die korrekte Handhabung gezeigt. Der Vorgang wird mehrmals wiederholt, auf spezielle Schwierigkeiten einzelner Applikationssysteme wird im Besonderen eingegangen. Am Ende des Programmes steht ein Quiz, durch das Lerninhalte überprüft und gefestigt werden können. Ungeschulte Gruppen: Im Folgenden werden die Gruppen 1, 3, 4, 6a als ungeschulte Gruppen zusammengefasst. Geschulte Gruppen: Als geschulte Gruppen werden die Gruppen $2,5,6$ busammengefasst.

die wesentlichen Handhabungsschritte in Punktwerten abbildete und so eine direkte Vergleichbarkeit ermöglichte. Einzelheiten sind der Tab. 2 zu entnehmen.

Eine gesonderte Untersuchung befasste sich mit der Fehleranalyse durch Dosier-Aerosol-Phantom (Vitalograph) und TurbohalerPhantom (Astra/Zeneca) an jeweils 23 Patienten. Die Bewertung des Dosier-Aerosol-Phantoms erfolgte nach den Kriterien Sprühstoßabgabe, Abgabephase, Einatemphase, Anhaltephase. Eine Anzeige der einzelnen Schritte am Display zeigt die korrekte oder falsche Durchführung. Die Beurteilung der Applikation durch das Turbohaler-Phantom erfolgte entsprechend Tab.3. Der Schritt 4 wird in der Einatemtiefe durch maximal 3 Punkte bewertet.

Ein weiterer Teil der Untersuchung befasste sich mit der Prüfung des theoretischen Wissens. Den Patienten wurden 5 wichtige Schritte der Applikationshandhabung in zufälliger Reihenfolge vorgelegt. Die Aufgabe für die Patienten bestand darin, diese in eine richtige Reihenfolge zu bringen. Hierbei wurden 95 Patienten befragt. Beispielhaft ist in Tab. 3 die Vorgabe für den Turbohaler aufgeführt. Entsprechend wurden die Einzelschritte je nach Device adjustiert. Der Patient hatte die richtige Reihenfolge als Ziffernfolge einzutragen. 
Tab. 2 Ablauf inhalativer Applikation und zugeordneter Punktescore. Volle Punktzahl für den jeweiligen Schritt wurde bei korrekter Durchführung dieses Schrittes gegeben

\begin{tabular}{|c|c|}
\hline 1. öffnen & 1 Punkt \\
\hline 2. laden/schütteln & 2 Punkte \\
\hline 3. ausatmen (tief, schwach, nicht, durch DA) & 2 Punkte \\
\hline 4. Mundstück umschließen & 1 Punkt \\
\hline $\begin{array}{l}\text { 5.1. auslösen (bei tiefer Einatmung, schwacher Einatmung, } \\
\text { Ausatmung) } \\
\text { 5.2. und einatmen (bei Beginn, Mitte, Ende der Einatmung) }\end{array}$ & $\begin{array}{l}2 \text { Punkte } \\
2 \text { Punkte }\end{array}$ \\
\hline 6. Atem anhalten & 2 Punkte \\
\hline 7. ausatmen & 1 Punkt \\
\hline 8. schließen & 1 Punkt \\
\hline Summe & 14 Punkte \\
\hline
\end{tabular}

Tab. 3 Beispiel Handlungsablauf Turbohaler. Der Patient hat die Aufgabe, den Ablauf mit Zahlen in einer korrekten Reihenfolge zu ordnen

$\begin{array}{ll}5 & \text { Atem anhalten } \\ 4 & \text { einatmen durch den Turbohaler } \\ 2 & \text { laden (Dosierrad hin- und zurückdrehen) } \\ 1 & \text { offnen } \\ 3 & \text { ausatmen }\end{array}$

Zur Prüfung auf Signifikanz wurde die einfaktorielle Varianzanalyse (F-Test) durchgeführt. Als Signifikanzniveau wurde $<0,05$ definiert.

\section{Ergebnisse}

Die Abb.1 zeigt die Verteilung der Summenscores der Handhabungsfehler der Patientengruppen 1 bis 5 . Die geringsten Punktwerte fanden sich bei den Gruppen 3 und 4, also bei Patienten, die sich ausschließlich über den Beipackzettel informiert hatten oder bei Patienten ohne jegliche Information. Es stellte sich heraus, dass der Informationswert durch den Beipackzettel nicht besser ist als keinerlei Information. Sowohl Patienten mit DosierAerosol-Erfahrung und mehrfachen ambulanten Schulungen (Gruppe 2) wie auch Patienten mit Kleingruppenschulung auf der Station während der klinischen Rehabilitation, zeigten befriedigende Ergebnisse.

Die gesonderte Betrachtung der Gruppen 6a und 6b (Abb.1) zeigt den schlechten Handhabungsstand bei Dosieraerosol-naiven Patienten ohne Schulung (Gruppe 6a) und die dramatische Verbesserung dieser Patienten nach Schulung mittels PC-basiertem interaktiven Patiententraining „More-BREATH“. Patienten, die eine fehlerfreie Handhabung hatten, konnten von $28 \%$ auf $79 \%$ der Gesamtstichprobe erhöht werden.

Die Analyse ausgewählter Applikationsschritte bestätigt das Gesamtbild noch einmal deutlich. Der Schritt „Öffnen“ wurde in al-

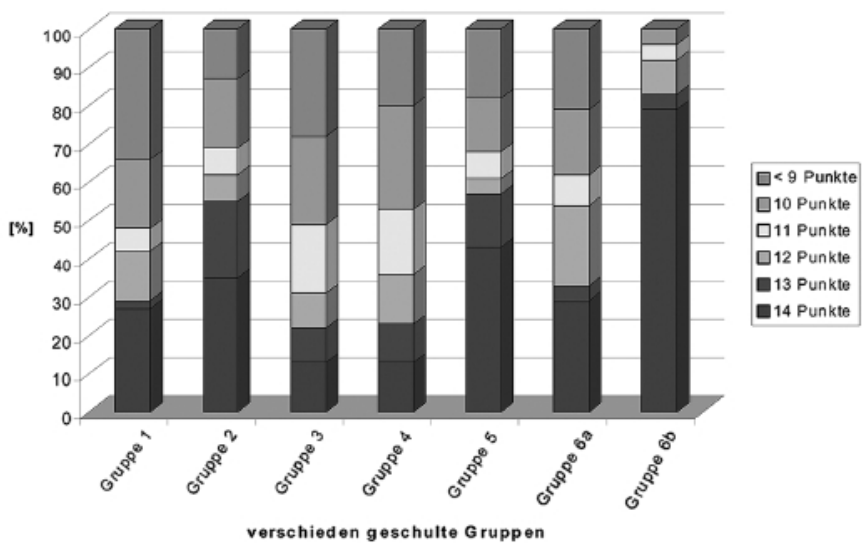

Abb. 1 Säulendarstellung der erreichten Punktwerte in Prozent der verschieden geschulten Gruppen.

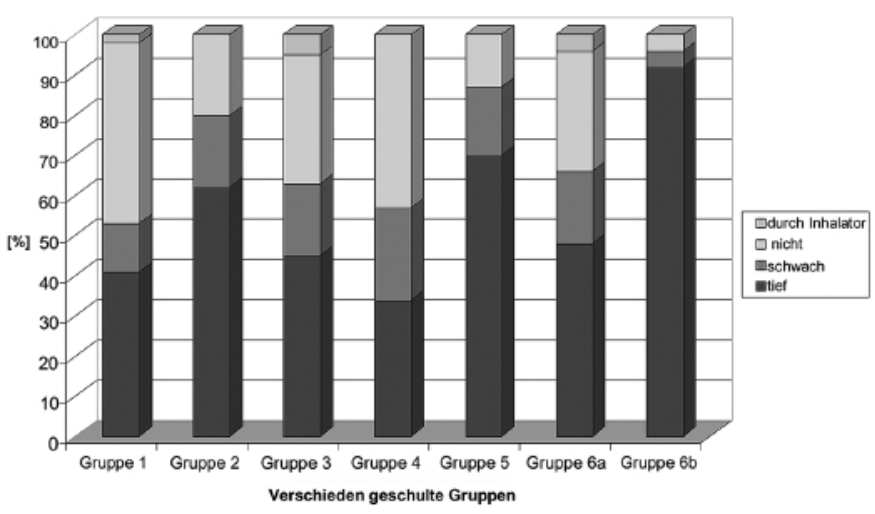

Abb. 2 Säulendarstellung der Mittelwerte in Prozent der verschieden geschulten Gruppen beim Schritt 3: Ausatmen vor der Inhalation.

len Gruppen vergleichbar richtig gemacht. Der Vorgang „Schütteln“, bzw. „Laden“ wurde meistens richtig durchgeführt. In den geschulten Gruppen 2, 5, 6b in über 90\% der Fälle, in den ungeschulten Gruppen 1, 3, 4 und 6a unter $90 \%$.

Die gesonderte Darstellung des Vorgangs „Ausatmen vor Inhalation“ (Abb. 2) war am schlechtesten in den Gruppen 3 und 4, also bei Dosier-Aerosol-naiven Patienten mit Beipackzettelinformation oder ohne jegliche Information. Patienten mit Dosier-AerosolErfahrung und mehreren ambulanten Schulungen (Gruppe 2), sowie Patienten mit spezieller Kleingruppenschulung auf der Station (Gruppe 5) schnitten deutlich besser ab.

Auffallend ist die Steigerung der korrekten Handhabung in der zunächst Dosier-Aerosol-naiven Gruppe 6a im Vergleich nach der Schulung mit PC-basiertem Programm als Gruppe 6b.

Ausatmen durch den Applikator wurde nur in den nichtgeschulten Gruppen 1, 3 und 6a festgestellt.

Der Handhabungsschritt „Mundstück umschließen“ wurde in allen untersuchten Gruppen zu über $90 \%$ richtig gemacht. Deutlich unterschiedlicher zeigten sich die Ergebnisse bei der Analyse des Schrittes „Inhalation“ (Abb.3); betrachtet man das Kriterium „tiefe Inhalation“ so schneiden wiederum die Gruppen der Dosier-Aerosol-naiven Patienten mit Beipackzettelinformation 


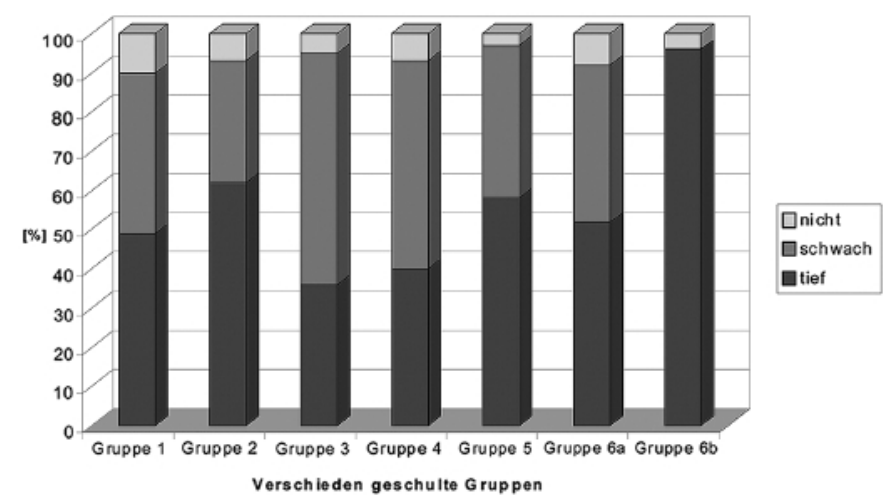

Abb. 3 Säulendarstellung der Mittelwerte in Prozent der verschieden geschulten Gruppen beim Schritt 5: Inhalation des Inhalates.

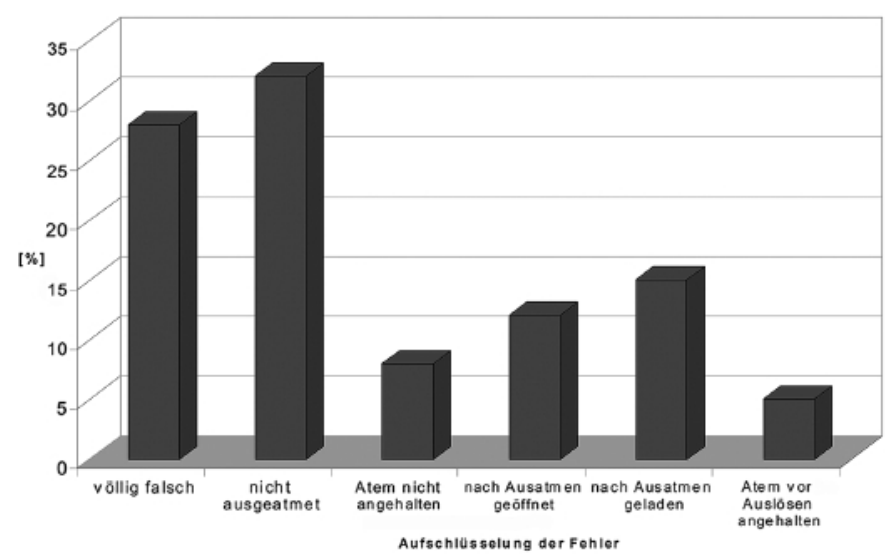

Abb. 4 Säulendarstellung der Mittelwerte der Fehlerhäufigkeit in Prozent.

(Gruppe 3) und die Patienten ohne jegliche Information (Gruppe 4) am schlechtesten ab. Herausragend sicher in der Applikation sind Patienten mit Erfahrung im Gebrauch und Schulungserfahrung (Gruppe 2) und insbesondere Patienten nach interaktiver PC-basierter Schulung (Gruppe 6b).

Ähnlich verhalten sich die Ergebnisse für die Schritte „Anhalten des Atmens nach Inhalation“, „Langsames Ausatmen nach der Inhalation“ und „Schließen des Applikators“. Das Anhalten des Atems wurde von $65 \%$ bis $100 \%$ richtig gemacht, stark abhängig von den Vorkenntnissen und der Schulungsart. Langsames Ausatmen und Schließen des Applikators wurde jeweils zu über $90 \%$ durchgeführt.

Die Überprüfung der Handhabung an Phantomen zeigte vor allem für das Druck-Dosier-Aerosol (Vitalograph) hohe Fehlerraten. Nur $13 \%$ der Patienten zeigten eine korrekte Inhalationsphase und nur $61 \%$ der Patienten hatten eine ausreichende Atemanhaltephase nach Applikation.

Die Patientengruppe, die das Turbohaler-Phantom benutzte, zeigte, dass nur 54\% der Patienten die Inspirationsphase optimal durchführten, das Ausatmen vor der Applikation und das Atemanhalten nach der Applikation machten 83\% der Patienten richtig.
Die Überprüfung der Handhabung der Phantome erfolgte aus einer zufälligen Stichprobe des Patientengutes, unabhängig von Gruppenzuteilungen.

Die Analyse des theoretischen Wissenstests (Abb.4) zeigte bei nur 58\% der Patienten eine völlig richtige Zuordnung der Reihenfolge. Die häufigsten Fehler bezogen sich auf die Punkte „nicht ausgeatmet vor Applikation“, „Gerät nach Ausatmen geladen“ und „Gerät nach Ausatmen geöffnet“.

\section{Diskussion}

Die Bedeutung der inhalativen Applikation in der Pharmakotherapie obstruktiver Atemwegserkrankungen ist eminent.

Gerade mit der zunehmenden Verbreitung hochwirksamer inhalativer Steroide und langwirkender Beta 2 Adrenergika sowie Anticholinergika konnte die inhalative Applikation auf $2 \times$ tgl., teilweise sogar $1 \times \operatorname{tgl}$. reduziert werden. In dem Maße, in dem die zu fordernde Inhalationshäufigkeit geringer wird, steigt die Bedeutung der „Treffsicherheit“ der Applikation.

Die hier vorgestellten Ergebnisse und die Ergebnisse von Voruntersuchungen $[1,3,10,12]$ zeigen die dramatische Häufung von Handhabungsfehlern bei Patienten, die nach der Verordnung sich selbst überlassen werden. In vorliegender Studie schnitten die Patienten am schlechtesten ab, die Dosier-Aerosol-naiv waren und keinerlei Information erhielten, sowie die Gruppe, die Dosier-Aerosol-naiv war und mittels Beipackzettel allein gelassen wurde. Hier lag die Quote in bezug auf das Kriterium „alles richtig gemacht" bei nur $13 \%$. Bei der Betrachtung der einzelnen Schritte zeigte sich, dass vor allem das tiefe Ausatmen vor der Inhalation, die folgende Inhalation und das Atemanhalten nach Applikation mit Fehlern behaftet waren. Bei Schulungen sollte daher ein besonderes Augenmerk auf die Vermittlung dieser Applikationsschritte gelegt werden. Applikationsschulung ist aus diesem Grunde ein Sonderschulungsmodul an der Fachklinik Bad Reichenhall [11] und kann die Compliance der Patienten in Hinblick auf die Applikation deutlich verbessern.

Die vorgestellten Ergebnisse belegen einen wesentlichen Effekt der PC-basierten interaktiven Schulung mittels CD-Rom („MoreBREATH“). Im Rahmen dieses Programms, das selbsterklärend im Beisein eines Lehrers durchlaufen wird, kann der Inhalationsvorgang für den gewünschten Applikatortyp beliebig oft beobachtet werden. Daneben wird auf spezifische Schwierigkeiten bei den jeweiligen Applikatoren hingewiesen und insbesondere auf Fehlermöglichkeiten und deren Vermeidung. Die Ergebnisse anhand der Applikatorphantome zeigen in spezieller Art die Fehlerhäufigkeit, die auftreten können, wobei beim Dosier-Aerosol wiederum die Koordination des Auslösens mit der Einatmung Schwierigkeiten bereitet hat. Patienten, die Koordinationsprobleme besitzen, sollten ggf. auf inspirationsaktivierte Pulverapplikatoren umgestellt werden.

Patienten perzeptieren ihren Wissensmangel in der Regel nicht, überschätzen ihre Kenntnis und unterschätzen den notwendigen Schulungsaufwand [5]. Die Gesamtsituation wird noch einmal bei der Prüfung des theoretischen Wissens dokumentiert. Nur 
55\% der Patienten konnten den Ablauf ihrer Applikation korrekt angeben.

Die praktischen Schlussfolgerungen aus diesen Untersuchungen zeigen erneut, dass der Kenntnisstand, wie auch die Fähigkeit der Handhabung bei inhalativer Medikamentenapplikation verbesserungsbedürftig ist. Die Untersuchung zeigt insbesondere den Wert von Schulungsmaßnahmen, die in Abhängigkeit von ihrer Intensität eine unterschiedliche Effizienz zeigen. Eine inhalative Pharmakotherapie bei obstruktiven Atemwegserkrankungen ohne adäquate Schulung ist unzureichend.

\section{Literatur}

${ }^{1}$ Beerendonk I, Mesters I, Mudde A et al. Assessment of the Inhalation Technique in Outpatients with Asthma or Chronic Obstructive Pulmonary Disease Using a Metered-Dose Inhalator or dry Powder Device. J Asthma 1998; 35: 273-279

${ }^{2}$ GOLD Global Initiative for Chronic Obstructive Lung Diseasae. Global strategy for diagnosis. management, and prevention of chronic obstructive pulmonary disease. NHLBL WHO Workshop Report. Executive Summary. National Institute of Health/National Heart, Lung and Blood institute

${ }^{3}$ Goodman D, Israel E, Rosenberg $\mathrm{M}$ et al. The Influence of Age, Diagnosis and Gender on Proper Use of Metered-Dose-Inhalers. Am J Respir Crit Care Med 1994; 5: 1256 - 1261

${ }^{4}$ Larson J, Hahn M, Ekholm B et al. Evaluation of Conventional Pressand-Breathe Metered-Dose-Inhaler Technique in 501 Patients. J Asthma 1994; 3: 193 - 199
${ }^{5}$ Petro W, Gebert P, Lauber B. Ursachenanalyse fehlerhafter Anwendung von Dosier-Aerosolen. Pneumologie 1994; 3: 191-196

${ }^{6}$ Schultz K, Schwiersch M, Petro W et al. Individualisiertes, modular strukturiertes Patientenverhaltenstraining bei obstruktiven Atemwegserkrankungen in der stationären Rehabilitation. Pneumologie 2000; 54: 296 - 305

${ }^{7}$ Serra-Battles J, Plaza V, Badiola C et al. Patient Perception and Acceptability of Multidose Dry powder Inhalers: A Randomized Crossover Comparison of Diskus/Accuhaler with Turbuhaler. J Aerosol Med 2002; 15: 59-64

${ }^{8}$ Shresta M, Parupia H, Andrews B et al. Metered-Dose-Inhaler Technique of Patients in an Urban ED: Prevalence of Incorrect Technique and Attempt at Education. Am J Emerg Med 1996; 4: 380 - 383

${ }^{9}$ Spohn S, Wittmann M, Petro W. Patientenverhaltenstraining bei COPD: Konzepte und Effizienz. Pneumologie 2000; 54: 72 - 79

${ }^{10}$ Sprossmann A, Kutschka F, Enk M et al. Beeinflussende Faktoren zum korrekten Gebrauch von Dosier-Aerosolen. Z Erkr Atmungsorgane 1991; 4-5: $93-95$

${ }^{11}$ Stark HJ, Schultz K, Petro W. Neue Wege in der Patientenschulung, Entwicklung PC-basierter Schulungsprogramme als Komponente des Patientenverhaltenstrainings. In: Petro W (Hrsg). „Patientenverhaltenstraining bei obstruktiven Atemwegserkrankungen Formen-Inhalte-Ergebnisse-Zukunft“. Dustri-Verlag Dr. Karl Feistle

12 Tan NC, Ng CJ, Goh S et al. Assessment of Metered Dose Inhaler Technique in Family Health Service Patients in Sinapore. Singapore Med J 1999; 40: $465-467$

${ }^{13}$ Wettengel R, Berdel D, Hofmann D et al. Asthmatherapie bei Kindern und Erwachsenen. Med Klin 1998; 11: 639-650

14 Worth $\mathrm{H}$ et al. Leitlinie der Deutschen Atemwegsliga und der Deutschen Gesellschaft für Pneumologie zur Diagnostik und Therapie von Patienten mit chronisch obstruktiver Bronchitis und Lungenemphysem (COPD). Pneumologie 2002; 56: 704-738 